\title{
Maximum Ratio Combining Precoding for Multi-Antenna Relay Systems
}

\author{
Hamid Reza Bahrami ${ }^{1}$, Tho Le-Ngoc ${ }^{2}$ \\ ${ }^{1}$ Department of ECE, University of Akron, Akron, USA \\ ${ }^{2}$ Department of ECE, McGill University, Montreal, Canada \\ E-mail: tho.le-ngoc@mcgill.ca \\ Received November 16, 2009; revised January 30, 2010; accepted February 28, 2010
}

\begin{abstract}
This paper addresses the design of practical communication strategies for multi-antenna amplify-and-forward and decode-and-forward relay systems. We show that simple linear techniques at the source and destination in conjunction with maximum ratio combining can provide an optimal transmission strategy in terms of received SNR without imposing a huge computational load over the relay node(s). Besides, the structures of precoding matrices are very similar at the source and relay nodes, which reduce the complexity as all nodes can play the role of source and relay nodes without changing their transmission structure. Numerical results show that the proposed transmission and reception techniques can improve the received SNR, and hence enhance the ergodic capacity.
\end{abstract}

Keywords: Cooperation, Relay System, Linear Precoding, Maximum Ratio Combining

\section{Introduction}

Relay networking [1-5] is one of the frameworks in which the concept of cooperation [6] becomes meaningful. In these systems, a source node tries to send its corresponding information to the destination node with the help of one or a number of relay node(s). Cooperative relaying targets additional diversity and coding gain and provides additional level of reliability, particularly when the direct source-destination link has poor quality.

Two natural questions in the context of relay networks are the problems of transmission and reception strategies. Mainly, how the source and relay node(s) should send the information to the destination node and how the destination should optimally combine the source information with replicate version(s) of information from relay node(s). Obviously, one cannot answer to these two questions separately. In other words, transmission and reception strategies should be jointly optimized.

A major practical issue to be addressed in the design of transmission and reception schemes in relay networks is complexity. Unlike point-to-point transmission schemes in which transmitter and receiver are responsible for the recovery of their own information, in the relay systems, other parts of the network are also engaged in the communications. Hence, it is very desirable that the communication strategy imposes minimum level of com- putational loads on the relay nodes. This point becomes one of the constraints that should be taken into account in the design of strategies for relay systems.

Two popular strategies can be considered for transmission in relay networks: amplify-and-forward (AF), in which a relay node does not decode the received signal but forwards it to the destination with a specific weight (e.g., [5,7-10]), and decode-and-forward (DF), in which a relay node decodes the received signal from the source and retransmits a decoded version of the signal to the destination (e.g., [11,12]). In this paper, we develop practical transmission and reception for both cases. In addition, throughout this paper, we assume that all terminals (nodes) operate in a half-duplex mode.

While there is a vigorous body of work on the relay systems in which each individual terminal is equipped with single antenna, the case of multi-antenna nodes has not been studied extensively. In [13], it was shown that the relay systems with MIMO capability offer a promising capacity and this capacity scales linearly with the number of antennas at source/destination and logarithmically with the number of relay nodes or antennas. For a similar case, a cooperative beamforming approach that can achieve the capacity of the network in the limit of large number of relay nodes was proposed in [14]. Another interesting setup can be found in [15] where the authors elaborate the effect of relay-assisted transmission 
on the capacity of rank-deficient MIMO systems.

While all the above results are attractive from a theoretical point of view, the need for practical transmission and reception schemes that can practically realize the ability of multi-antenna relay networks in providing higher capacity and performance compared to that of systems with single-antenna terminals is still pronounced. In [16], three signaling strategies for multi-antenna relay systems are discussed and compared. An optimal hybrid relaying strategy based on a combination of filtering and AF protocol was derived and can outperform AF relaying, especially when Channel State Information (CSI) is available at the relay node. In [17], an AF relaying structure that maximizes the capacity when there is no direct link between source and destination nodes has been introduced. The authors, however, mentioned that their analysis is intractable when there is a direct link between source and destination nodes.

The scarcity of studies on the design of communication schemes for multi-antenna relay systems in the literature is the main motivation of this study. More specifically, throughout this paper, we assume the source, destination and relay nodes are all equipped with multiple antennas. Our goal is to find optimal transmission and reception schemes for this setup while avoiding a huge complexity especially at the relay node. We show that a maximum ratio combining scheme at the receiver in conjunction with suitable linear precoding techniques at transmit and relay node can lead us to this end. Our study shows that the proposed scheme is optimal in terms of received SNR (and capacity) while maintaining an acceptable computational load at all nodes. In addition, the technique can be applied to both AF and DF protocols with small modifications. This feature can facilitate switching between two protocols whenever necessary. On the other hand, structures of source and relay nodes are identical, and, hence, enable a node to play the role of the source or relay in different time instants without the need of additional software or hardware overhead.

We further show that a Generalized Maximum Ratio Combiner (GMRC) at the destination is optimum for both AF and DF protocols in terms of SNR. Furthermore, for DF protocol, the precoders at the source and relay nodes should send the information in the direction of the eigenvectors corresponding to the strongest eigenvalues of the channel matrices. While it is straightforward to derive the precoding structure in the case of DF protocol, the case of AF cannot be elaborated easily. We instead propose a relay selection scheme that can result in the best possible received SNR.

The rest of the paper is organized as follows. In Section 2, we present the system model of the multi-antenna relay network. In Section 3, maximum ratio combining schemes for different scenarios such as point-to-point MIMO, multipoint-to-point system, AF and DF relaying are studied. Section 4 is allocated to the precoder design for transmit and relay nodes and in Section 5, numerical results are presented. Conclusions are given in Section 6.

\section{System Model}

We consider a relay system composed of one $M$-antenna source (transmit) node, one $L$-antenna relay node and one $\mathrm{N}$-antenna destination (receive) node, operating in a half-duplex mode. For simplicity in notations, in the following analysis, we assume $L=M$ and single-symbol transmission, i.e., at a specific time instant, the source tends to transmit a symbol $x$ of a pre-determined code book (or constellation) to the destination ${ }^{1}$. It applies a precoding vector $\mathbf{w}_{1}$ of size $M \times 1$ to this symbol and sends it to both the destination and relay nodes. In the next time slot, based on the specific protocol (AF or DF), the relay node multiplies the received symbol by another precoding vector $\mathbf{w}_{2}$ of the same size and resends this precoded version to the destination. Destination then combines the two received signals based on a maximum-ratio-combining strategy.

The received signal in the first time slot can be written as:

$$
\mathbf{y}_{1}=\sqrt{\gamma_{1}} \mathbf{H}_{1} \mathbf{w}_{1} x+\mathbf{n}_{1}
$$

where $\mathbf{H}_{1}$ is the $N \times M$ forward channel matrix with normalized circularly symmetric Gaussian random entries, $\gamma_{1}$ is its corresponding SNR, $\mathbf{y}_{1}$ and $\mathbf{n}_{1}$ are the received and white Gaussian noise vectors of size $N \times 1$, respectively. In the second time slot, the received signal is:

$$
\mathbf{y}_{2}=\sqrt{\gamma_{2}} \mathbf{H}_{2} \mathbf{w}_{2} \tilde{x}+\mathbf{n}_{2}
$$

where $\tilde{x}$ is either a detected version of $x$ at relay node for DF or $\tilde{x}=\sqrt{\gamma_{G}} \mathbf{G w}_{1} x+\mathbf{n}$ for AF scenario. $\mathbf{G}$ is the source-relay $M \times M$ channel matrix, $\mathbf{n}$ is the $M \times 1$ noise vector at relay and $\gamma_{G}$ is its corresponding SNR. $\mathbf{H}_{2}, \gamma_{2}$, $\mathbf{y}_{2}$ and $\mathbf{n}_{2}$ are defined similar to their counterparts in (1). The destination combines these two signals using two weight vectors $\tilde{\mathbf{w}}_{1}$ and $\tilde{\mathbf{w}}_{2}$ to construct the received signal

$$
y=\tilde{\mathbf{w}}_{1}^{H} \mathbf{y}_{1}+\tilde{\mathbf{w}}_{2}^{H} \mathbf{y}_{2}
$$

The decision is made on $y$ to detect the transmit symbol $x$. Our goal is to find two precoding $\left(\mathbf{w}_{1}\right.$ and $\left.\mathbf{w}_{2}\right)$ and two weight vectors $\left(\tilde{\mathbf{w}}_{1}\right.$ and $\left.\tilde{\mathbf{w}}_{1}\right)$ such that the received SNR is maximized. For limited transmit power, $\mathbf{w}_{1}$ and $\mathbf{w}_{2}$ are assumed to be

$$
\mathbf{w}_{1}^{H} \mathbf{w}_{1}=\left\|\mathbf{w}_{1}\right\|^{2} \leq 1 \quad \mathbf{w}_{2}^{H} \mathbf{w}_{2}=\left\|\mathbf{w}_{2}\right\|^{2} \leq 1
$$

${ }^{1}$ It is not difficult to generalize the discussions to the cases of $L \neq M$ and multiple-symbol transmission. For multiple-symbol transmission, the following analysis depends on the coding used to map the multiple symbols over multiple antennas, e.g., space-time coding, and one should also assume that $N \geq L \geq M$ so that destination (and relay) nodes are able to detect all transmitted symbols. 
Maximizing SNR will minimize the probability of wrong decision over $x$. Also, we show that maximizing SNR in this scenario is equivalent to maximizing the instantaneous mutual information and ultimately the system capacity.

\section{Generalized Maximum Ratio Combining}

\subsection{DF Relay-Assisted MIMO System}

We first start consider a point-to-point MIMO system with $M$ transmit antennas and $N$ receive antennas in Figure 1(a). The transmitted symbol $x$ is precoded at transmitter by precoder $\mathbf{w}$ and the received vector is combined using vector $\tilde{\mathbf{w}}$ at the receiver. Therefore, the system model can be written as:

$$
\mathbf{y}=\sqrt{\gamma} \tilde{\mathbf{w}}^{H} \mathbf{H w} x+\tilde{\mathbf{w}} \mathbf{n}
$$

where $\mathbf{H}, \mathbf{n}$ and $\gamma$ are similar to $\mathbf{H}_{1}$ and $\mathbf{n}_{1}$ in (1), and the corresponding received SNR is

$$
\mathbf{S N R}=\gamma \frac{\mathbf{w}^{H} \mathbf{H}^{H} \tilde{\mathbf{w}} \tilde{\mathbf{w}}^{H} \mathbf{H w}}{\tilde{\mathbf{w}}^{H} \tilde{\mathbf{w}}}
$$

It is well known that one can select $\tilde{\mathbf{w}}=\mathbf{H w}$ to achieve the maximum SNR $=\gamma \mathbf{w}^{H} \mathbf{H}^{H} \mathbf{H w}$ and maximum mutual information between $x$ and $\tilde{x}$,

$$
I(x ; \tilde{x})=\log (1+\mathrm{SNR})=\log \left(1+\gamma \mathbf{w}^{H} \mathbf{H}^{H} \mathbf{H w}\right) .
$$

Now let consider the case when two transmitters send the same information to a receiver but in different time instants as shown in Figure 1(b). Both transmitters are equipped with $M$ antennas while the receiver has $N$ antennas. This case also corresponds to an ideal DF scenario when the relay node can always correctly decode the source information. The system model is the same as (1) and (2) except that $\tilde{x}$ is replaced by $x$ in (2). Combining (1), (2) and (3), one can write an equivalent compound system equation for the above scenario as

$$
y=\left(\tilde{\mathbf{w}}_{1}^{H} \tilde{\mathbf{w}}_{2}^{H}\right)\left(\begin{array}{cc}
\sqrt{\gamma_{1}} \mathbf{H}_{1} & \mathbf{0} \\
\mathbf{0} & \sqrt{\gamma_{2}} \mathbf{H}_{2}
\end{array}\right)\left(\begin{array}{l}
\mathbf{w}_{1} \\
\mathbf{w}_{2}
\end{array}\right) x+\left(\tilde{\mathbf{w}}_{1}^{H} \tilde{\mathbf{w}}_{2}^{H}\right)\left(\begin{array}{l}
\mathbf{n}_{1} \\
\mathbf{n}_{2}
\end{array}\right)(6)
$$

This is clearly a MIMO system with $2 M$ transmit antennas and $2 N$ receive antennas, and the generalized maximum ratio combining (GMRC) scheme for such a system is

$$
\tilde{\mathbf{w}}=\mathbf{H}^{\prime} \mathbf{w}, \tilde{\mathbf{w}}_{1}=\mathbf{H}_{1} \mathbf{w}_{1} \text { and } \tilde{\mathbf{w}}_{2}=\mathbf{H}_{2} \mathbf{w}_{2}
$$

where

$$
\mathbf{H}^{\prime}=\left(\begin{array}{cc}
\sqrt{\gamma_{1}} \mathbf{H}_{1} & \mathbf{0} \\
\mathbf{0} & \sqrt{\gamma_{2}} \mathbf{H}_{2}
\end{array}\right) y ; \tilde{\mathbf{w}}=\left(\begin{array}{c}
\tilde{\mathbf{w}}_{1} \\
\tilde{\mathbf{w}}_{2}
\end{array}\right) \text { and } \mathbf{w}=\left(\begin{array}{c}
\mathbf{w}_{1} \\
\mathbf{w}_{2}
\end{array}\right)
$$

Note that there is a fundamental difference between MRC and GMRC as MRC just considers combining in space domain over elements of receive antennas while GMRC includes combining over both space and time.

In a general DF scenario, the received signal after combining becomes

$$
y=\left(\tilde{\mathbf{w}}_{1}^{H} \tilde{\mathbf{w}}_{2}^{H}\right)\left(\begin{array}{cc}
\sqrt{\gamma_{1}} \mathbf{H}_{1} & \mathbf{0} \\
\mathbf{0} & \sqrt{\gamma_{2}} \mathbf{H}_{2}
\end{array}\right)\left(\begin{array}{c}
\mathbf{w}_{1} x \\
\mathbf{w}_{2} x^{\prime}
\end{array}\right)+\left(\tilde{\mathbf{w}}_{1}^{H} \tilde{\mathbf{w}}_{2}^{H}\right)\left(\begin{array}{l}
\mathbf{n}_{1} \\
\mathbf{n}_{2}
\end{array}\right)(8)
$$

where $x^{\prime}$ denotes the symbol decoded and re-transmitted by the relay, i.e., $x^{\prime}=x$ for correct detection and $x^{\prime} \neq x$ for erroneous detection. Let $\alpha$ denote the probability of erroneous detection at the relay. The optimum combining vectors for DF transmission can be written as:

$$
\tilde{\mathbf{w}}_{1}=\mathbf{H}_{1} \mathbf{w}_{1} \text { and } \tilde{\mathbf{w}}_{2}=\delta \mathbf{H}_{2} \mathbf{w}_{2}
$$

where the coefficient $\delta$ can be estimated from $\alpha$. Consider an approximation by assuming that $x^{\prime}=-x$ for an erroneous detection at the relay. Note that this consideration is exact for binary transmission. For a general M-ary signaling scheme, this assumption represents a pessimistic consideration. Under this assumption, the SNR's corresponding to the cases of correct and erroneous detection at the relay are respectively,

$$
\mathrm{SNR}_{1}=\frac{\left(d_{1}+d_{2}\right)\left(d_{1}^{\prime}+d_{2}^{\prime}\right)}{d_{o}}, \mathrm{SNR}_{2}=\frac{\left(d_{1}-d_{2}\right)\left(d_{1}^{\prime}-d_{2}^{\prime}\right)}{d_{o}},
$$

where

$$
\begin{gathered}
d_{1}=\sqrt{\gamma_{1}} \mathbf{w}_{1}^{H} \mathbf{H}_{1}^{H} \tilde{\mathbf{w}}_{1}, d_{2}=\sqrt{\gamma_{2}} \mathbf{w}_{2}^{H} \mathbf{H}_{2}^{H} \tilde{\mathbf{w}}_{2}, d_{1}^{\prime}=\sqrt{\gamma_{1}} \tilde{\mathbf{w}}_{1}^{H} \mathbf{H}_{1} \mathbf{w}_{1}, \\
d_{2}=\sqrt{\gamma_{2}} \tilde{\mathbf{w}}_{2}^{H} \mathbf{H}_{2} \mathbf{w}_{2} \text {, and } d_{o}=\tilde{\mathbf{w}}_{1}^{H} \tilde{\mathbf{w}}_{1}+\tilde{\mathbf{w}}_{2}^{H} \tilde{\mathbf{w}}_{2} .
\end{gathered}
$$

The average received SNR can then be written as (11).

From (11) and considering a system equation similar to (6) and (7), one can find the corresponding equivalent system equation for this general DF scenario as (12).

$$
\begin{aligned}
& \mathrm{SNR}=(1-\alpha) \mathrm{SNR}_{1}+\alpha \mathrm{SNR}_{2}= \\
& \frac{\left(\gamma_{1} \mathbf{w}_{1}^{H} \mathbf{H}_{1}^{H} \tilde{\mathbf{w}}_{1} \tilde{\mathbf{w}}_{1}^{H} \mathbf{H}_{1} \mathbf{w}_{1}+\gamma_{2} \mathbf{w}_{2}^{H} \mathbf{H}_{2}^{H} \tilde{\mathbf{w}}_{2} \tilde{\mathbf{w}}_{2}^{H} \mathbf{H}_{2} \mathbf{w}_{2}\right)+(1-2 \alpha) \sqrt{\gamma_{1} \gamma_{2}}\left(\mathbf{w}_{1}^{H} \mathbf{H}_{1}^{H} \tilde{\mathbf{w}}_{1} \tilde{\mathbf{w}}_{2}^{H} \mathbf{H}_{2} \mathbf{w}_{2}+\tilde{\mathbf{w}}_{1}^{H} \mathbf{H}_{1} \mathbf{w}_{1} \tilde{\mathbf{w}}_{2}^{H} \mathbf{H}_{2} \mathbf{w}_{2}\right)}{\tilde{\mathbf{w}}_{1}^{H} \tilde{\mathbf{w}}_{1}+\tilde{\mathbf{w}}_{2}^{H} \tilde{\mathbf{w}}_{2}}
\end{aligned}
$$

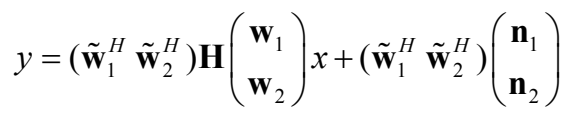

where $\mathbf{H}$ is the channel matrix of the equivalent MIMO system and can be approximated as:

$$
\mathbf{H} \approx\left(\begin{array}{cc}
\sqrt{\gamma_{1}} \mathbf{H}_{1} & \mathbf{0} \\
\mathbf{0} & \sqrt{\gamma_{2}}(1-2 \alpha) \mathbf{H}_{2}
\end{array}\right)
$$




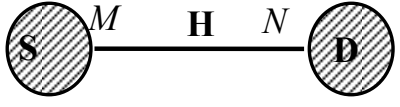

(a)

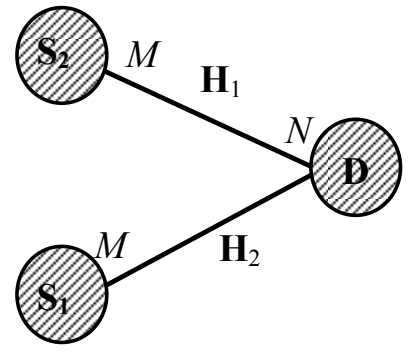

(b)

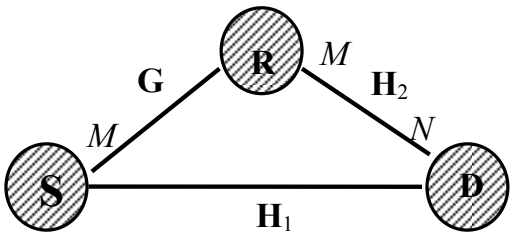

(c)

Figure 1: Diagrams of different transmission systems: (a) point-to-point MIMO; (b) multipoint-to-point MIMO; (c) relay-assisted MIMO.

In other words, the coefficient $\delta$ can be approximated as $\delta \approx 1-2 \alpha$ when $\alpha$ is small. Note that one can use other estimations to find $\delta$ or the optimum weight ad-hoc. In general, the idea is to reduce the weight of the signal in the second transmit interval as it may contain error and degrade the total received SNR at the destination.

\subsection{AF Relay-Assisted MIMO System}

The difficulty with this scenario is that the noise in the second time slot is no longer white. In other word, based on (1) and (2), the system equation in this case can be rewritten as:

$$
\begin{array}{r}
y=\left(\tilde{\mathbf{w}}_{1}^{H} \tilde{\mathbf{w}}_{2}^{H}\right)\left(\begin{array}{cc}
\sqrt{\gamma_{1}} \mathbf{H}_{1} & \mathbf{0} \\
\mathbf{0} & \sqrt{\gamma_{2} \gamma_{G}} \mathbf{H}_{2}
\end{array}\right)\left(\begin{array}{c}
\mathbf{w}_{1} \\
\mathbf{W}_{2}^{H} \mathbf{G} \mathbf{w}_{1}
\end{array}\right) x \\
+\left(\tilde{\mathbf{w}}_{1}^{H} \tilde{\mathbf{w}}_{2}^{H}\right)\left(\begin{array}{c}
\mathbf{n}_{1} \\
\mathbf{n}_{2}+\sqrt{\gamma_{2}} \mathbf{H}_{2} \mathbf{W}_{2}^{H} \mathbf{n}
\end{array}\right)
\end{array}
$$

The difference between the AF and DF protocols is that $\mathbf{W}_{2}$ is the $M \times M$ precoding matrix (instead of vector) at the relay node, and the noise in the second time interval is no longer white. Assuming $\mathbf{n}$ and $\mathbf{n}_{2}$ are uncorrelated, the autocorrelation function of the noise in the second time interval can be written as:

$$
\begin{gathered}
\boldsymbol{\Lambda}=E\left\{\left(\mathbf{n}_{2}+\sqrt{\gamma_{2}} \mathbf{H}_{2} \mathbf{W}_{2}^{H} \mathbf{n}\right)\left(\mathbf{n}_{2}+\sqrt{\gamma_{2}} \mathbf{H}_{2} \mathbf{W}_{2}^{H} \mathbf{n}\right)^{H}\right\} \\
=\mathbf{I}_{M}+\gamma_{2} \mathbf{H}_{2} \mathbf{W}_{2}^{H} \mathbf{W}_{2} \mathbf{H}_{2}^{H}
\end{gathered}
$$

where $\mathbf{I}_{M}$ denotes identity matrix of size $M$. Applying an eigenvalue decomposition over $\boldsymbol{\Lambda}$, one can write $\boldsymbol{\Lambda}=$ $\mathbf{U D U}^{H}$ where $\mathbf{U}$ and $\mathbf{D}$ are $N \times N$ unitary and diagonal matrices, respectively.

Defining the pre-whitening filter as $\mathbf{W}_{P}=\mathbf{D}^{-1 / 2} \mathbf{U}^{H}$ and applying it to the received signal in the second time-slot will make the output noise white. With that, one can apply (8) directly to derive the structure of combining vectors. The system model can be considered as (12) with:

$$
\mathbf{H}=\left(\begin{array}{cc}
\sqrt{\gamma_{1}} \mathbf{H}_{1} & \mathbf{0} \\
\mathbf{0} & \sqrt{\gamma_{2} \gamma_{G}} \mathbf{D}^{-1 / 2} \mathbf{U}^{H} \mathbf{H}_{2}
\end{array}\right)
$$

Now from (13), the optimum combining vectors for an AF relay-assisted MIMO system can be written as:

$$
\tilde{\mathbf{w}}_{1}=\mathbf{H}_{1} \mathbf{w}_{1} \text { and } \tilde{\mathbf{w}}_{2}=\mathbf{D}^{-1 / 2} \mathbf{U}^{H} \mathbf{H}_{2} \mathbf{W}_{2}^{H} \mathbf{G} \mathbf{w}_{1}
$$

In other words, the optimum weight vector in the second time interval is a combination of an MRC vector and a pre-whitening filter. By applying pre-whitening filter, the output noise will be white and therefore one can apply the combining weight vectors in (15) to maximize the SNR, the instantaneous mutual information and ultimately the system capacity.

\section{Precoding for Relay-Assisted MIMO Systems}

Our goal here is to investigate the design of precoding vectors, $\mathbf{w}_{1}$ and $\mathbf{w}_{2}$, in (1) and (2). We start with pointto-point MIMO transmission and then generalize the results to the case of relay-assisted MIMO systems.

Recall that, after applying MRC weight vector, (6) yields SNR $=\gamma \mathbf{w}^{H} \mathbf{H}^{H} \mathbf{H w}$. To maximize this SNR subject to power constraint over $\mathbf{w}$, similar to (4), one should take $\mathbf{w}$ in the direction of the eigenvector of Hermitian matrix $\mathbf{H}^{H} \mathbf{H}$ associated with $\lambda_{\max }$ where $\lambda_{\max }$ is the largest eigenvalue of $\mathbf{H}^{H} \mathbf{H}$. In other words, under the total transmit power constraint at source, the optimum precoding vector that maximizes the SNR of an MRC-based MIMO system can be written as

$$
\mathbf{w}=\mathbf{u}_{\max }\left(\mathbf{H}^{H} \mathbf{H}\right)
$$

where $\mathbf{u}_{\max }$ stands for the eigenvector of $\mathbf{H}$ corresponding the maximum eigenvalue. With this precoding vector and considering the receive combining vector of $\tilde{\mathbf{w}}=\mathbf{H w}$, the receive SNR of the system can be written as $\mathrm{SNR}=\gamma \lambda_{\max }$. From (8), (9) and (16), it can be shown that 
under the total transmit power constraints at source and relay nodes, for a multipoint-to-point MIMO system with GMRC combining vectors at receiver, the optimum precoding vectors are:

$$
\mathbf{w}_{1}=\mathbf{u}_{\max }\left(\mathbf{H}_{1}^{H} \mathbf{H}_{1}\right) \text { and } \mathbf{w}_{2}=\mathbf{u}_{\max }\left(\mathbf{H}_{2}^{H} \mathbf{H}_{2}\right)
$$

The same conclusion is also valid for the case of DF protocol for relay-assisted MIMO systems.

Now, we are ready to revisit the problem of AF protocol. The difficulty with this case is that the optimization of precoding matrix at the relay node, $\mathbf{W}_{2}$, is not independent of the optimization of precoding vector at the source node, $\mathbf{w}_{1}$. This is because the received SNR equation resulted in the second transmit interval is

$$
\mathrm{SNR}_{2}=\sqrt{\gamma_{2} \gamma_{G}} \mathbf{w}_{1}^{H} \mathbf{G}^{H} \mathbf{W}_{2} \mathbf{H}_{2}^{H} \mathbf{H}_{2} \mathbf{W}_{2}^{H} \mathbf{G} \mathbf{w}_{1}
$$

and, therefore, is a function of both $\mathbf{w}_{1}$ and $\mathbf{W}_{2}$. Note that (18) comes from applying (15) to calculate the SNR in (6). Although direct maximization of (18) for $\mathbf{W}_{2}$ would be difficult, one can make a clever guess that if we select $\mathbf{W}_{2}$ such that it maximizes SNR over the source-relay link, $\mathbf{G}$, this can ultimately result in the maximization of the SNR in (18) over the entire link from source to destination. Therefore, assume that $\mathbf{W}_{2}$ can be written as

$$
\mathbf{W}_{2}=\mathbf{G} \mathbf{w}_{1} \hat{\mathbf{w}}_{2}^{H}
$$

where $\mathbf{G w}_{1}$ is responsible for maximizing the SNR at relay node while $\hat{\mathbf{w}}_{2}$ is an independent vector reserved for further optimization of precoding matrix at the relay node. Moreover, for maximizing the SNR over the relay-destination link, using (16), it can be shown that

$$
\hat{\mathbf{w}}_{2}=\mathbf{u}_{\max }\left(\mathbf{H}_{2}\right)
$$

Now, $\mathbf{w}_{1}$, the precoding vector at the source node is the only remaining parameter to be selected. However, $\mathbf{w}_{1}$ affects the received SNR from both direct and relayed links. Therefore, to optimize $\mathbf{w}_{1}$, the SNR equations similar to (10) should be considered, which makes the optimization problem very difficult if not impossible to solve. To resolve this problem, we focus on each of the transmit intervals, separately. Since in a communication system, there are usually a number of available relay terminals (rather than just one), we, ultimately, propose the use of relay selection approach to maximize the overall SNR of the system.

Consider the received SNR in the first transmit interval:

$$
\mathrm{SNR}_{1}=\gamma_{1} \mathbf{w}_{1}^{H} \mathbf{H}_{1}^{H} \mathbf{H}_{1} \mathbf{w}_{1}
$$

Based on (16), $\mathrm{SNR}_{1}$ is maximized if $\mathbf{w}_{1}=\mathbf{u}_{\max }\left(\mathbf{H}_{1}\right)$. On the other hand, in the second transmit interval, by substituting $\mathbf{W}_{2}$ of (19) into (18), it turns out that the source precoding vector will be responsible for the source-relay portion of the SNR. Therefore, to maximize this portion, one should select $\mathbf{w}_{1}=\mathbf{u}_{\max }(\mathbf{G})$. These two equations for $\mathbf{w}_{1}$ are definitely in contrast with each other. The best scenario is that $\mathbf{u}_{\max }\left(\mathbf{H}_{1}\right)=\mathbf{u}_{\max }(\mathbf{G})$. In this case, based on (16), the overall receive SNR of the system can be written as:

$$
\mathrm{SNR}=\lambda_{\max }\left(\mathbf{H}_{1}^{H} \mathbf{H}_{1}\right)\left(\gamma_{1}+\gamma_{G} \gamma_{2} \lambda_{\max }\left(\mathbf{H}_{2}^{H} \mathbf{H}_{2}\right)\right)
$$

Now, let assume that $\mathbf{u}_{\text {max }}\left(\mathbf{H}_{1}\right) \neq \mathbf{u}_{\text {max }}(\mathbf{G})$ but there are $K$ available relay nodes in the system. The best relay should be selected such that the overall receive SNR is maximized. The performance degradation appears as a factor of $\left\langle\mathbf{u}_{\max }\left(\mathbf{H}_{1}\right), \mathbf{u}_{\max }(\mathbf{G})\right\rangle$ in the SNR equation. Two following extreme cases can be considered.

In the first case when the source-destination link is very strong (i.e., asymptotic case of $\lambda_{1} \rightarrow \infty$ ), one should choose the precoding vector in the direction of $\mathbf{u}_{\max }\left(\mathbf{H}_{1}\right)$. Therefore, the SNR loss as compared to the optimum case in (21), is due to the eigen-mismatch in the second transmit interval and the overall SNR can be expressed as:

$$
\begin{gathered}
\mathrm{SNR}=\lambda_{\text {max }}\left(\mathbf{H}_{1}^{H} \mathbf{H}_{1}\right) \gamma_{1}+ \\
\left\langle\mathbf{u}_{\text {max }}\left(\mathbf{H}_{1}\right), \mathbf{u}_{\text {max }}(\mathbf{G})\right\rangle \gamma_{G} \gamma_{2} \lambda_{\text {max }}\left(\mathbf{G}^{H} \mathbf{G}\right) \lambda_{\text {max }}\left(\mathbf{H}_{2}^{H} \mathbf{H}_{2}\right)
\end{gathered}
$$

In the second case when the relay links are much stronger compared to the direct link (i.e., asymptotic case of $\left.\lambda_{2}, \lambda_{G} \rightarrow \infty\right)$, the natural selection is the source precoding vector in the direction of $\mathbf{u}_{\max }(\mathbf{G})$. In this case, the SNR loss is due to the eigen-mismatch in the first transmit interval. The overall received SNR can also be written as:

$$
\begin{aligned}
\operatorname{SNR}=\left\langle\mathbf{u}_{\max }\left(\mathbf{H}_{1}\right), \mathbf{u}_{\max }(\mathbf{G})\right\rangle \lambda_{\text {max }}\left(\mathbf{H}_{1}^{H} \mathbf{H}_{1}\right) \gamma_{1}+ \\
\left.\gamma_{G} \gamma_{2} \lambda_{\max }\left(\mathbf{G}^{H} \mathbf{G}\right) \lambda_{\text {max }}\left(\mathbf{H}_{2}^{H} \mathbf{H}_{2}\right)\right)(23)
\end{aligned}
$$

Based on (22) and (23), amongst all $K$ candidate relay nodes, the best relay node can be selected as

$$
i=\arg \max _{i=1, \ldots, K}\left\langle\mathbf{u}_{\max }\left(\mathbf{H}_{1}\right), \mathbf{u}_{\max }(\mathbf{G})\right\rangle \gamma_{G} \gamma_{2} \lambda_{\max }\left(\mathbf{H}_{2}^{H} \mathbf{H}_{2}\right)
$$

if the source-destination link is stronger than the sourcerelay and relay-destination links, or

$$
\begin{aligned}
i=\arg \max _{i=1, \ldots, K} & \left\langle\mathbf{u}_{\max }\left(\mathbf{H}_{1}\right), \mathbf{u}_{\max }(\mathbf{G})\right\rangle \lambda_{\max }\left(\mathbf{H}_{1}^{H} \mathbf{H}_{1}\right) \\
& +\gamma_{G} \gamma_{2} \lambda_{\max }\left(\mathbf{H}_{2}^{H} \mathbf{H}_{2}\right)
\end{aligned}
$$

if the source-relay and relay-destination links are stronger than the source-destination link. $<,>$ stands for inner product. This selection maximizes the SNR of the relay-assisted AF system with high probability. In other words, the best relay is the one with strong source-relay and relay-destination links, i.e., large $\gamma_{G}$ and $\gamma_{2}$, and when the eigenvector corresponding to the maximum eigenvalue of source-relay link matrix is the closest to $\mathbf{u}_{\max }\left(\mathbf{H}_{1}\right)$. 


\section{Numerical Results}

We study the performance of the proposed MRC-based precoding technique by means of simulation. We consider all source, relay and destination nodes are equipped with two antennas, i.e., $M=N=2$. We investigate the received SNR and average mutual information for these systems.

Figure 2 shows the received SNR for different setups in Figure 1. For the sake of comparison, we also show the performance of the precoder based on equal gain combining in point-to-point MIMO transmission. First, we observe that MRC-based precoding (case $A$ ) outperforms precoding with equal gain combining in terms of received SNR by about $3 \mathrm{~dB}$. On the other hand, in the case of multipoint-to-point transmission (case $B$ ), pre- coding based on MRC can provide an additional gain as compared to the case of point-to-point transmission. This is mainly due to the availability of an additional path between the second transmit and receive nodes, which provides a higher average SNR at the receiver (especially when the link from first transmitter to receive node is poor). Finally, we see that the relay-assisted amplifyand-forward precoding system (case $C$ ) provides a received SNR comparable to that of an ideal multipoint-to-point system. The slight decrease in the received SNR is due to the noise accumulation in the relay node. The same conclusion is also valid for the case of a decode-and-forward system as there is a decrease in the received SNR due to the probability of wrong decision at the relay node.

Figure 3 also shows that the average mutual informa-

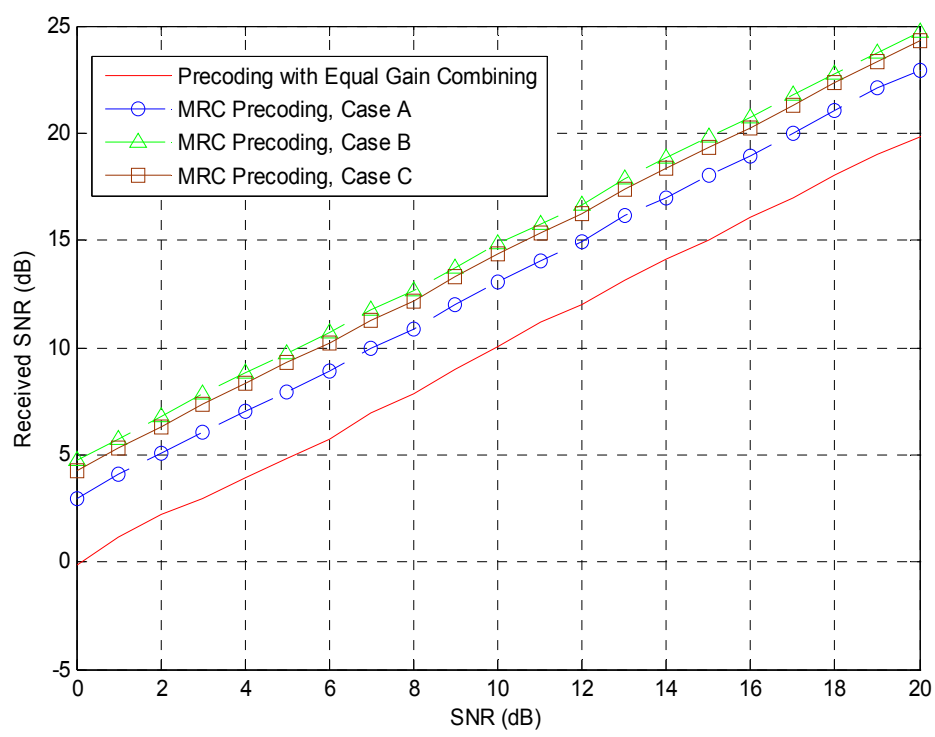

Figure 2. Received SNR in different scenarios.

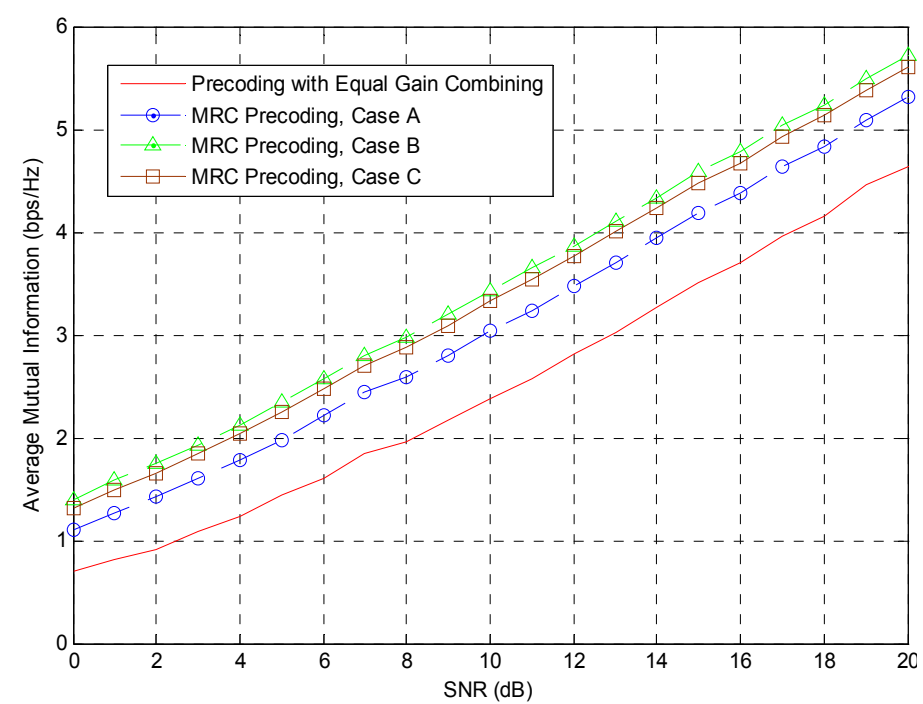

Figure 3. Average mutual information in different scenarios. 
tion for all the above scenarios. The same conclusions can be drawn for the average mutual information, i.e., the relay-assisted MIMO precoding can provide an average mutual information close to that in the case when two transmitters send identical information to the relay node.

\section{Conclusions}

We studied optimal linear transmit and receive strategies for a variety of MIMO systems. Our focus was mainly on relay-assisted MIMO systems. These systems are very attractive from both theoretical and practical points of view. However, there are still many open questions concerning transmission and reception schemes in the field. We built a framework based on the well-known MRC scheme on the receiver (destination) side. As we demonstrated, the construction of the optimal combining strategies for both DF and AF relaying protocols can be based on the concept of MRC.

We first derived the optimal linear receiver structure for these systems. Next, based on the structure of optimum receivers, we investigated the optimal linear precoding vectors for the source and relay nodes. Our results show that, for the optimum receiver, the optimal transmit precoding strategy for DF protocol is to send the information in the direction of the eigenvectors of the direct $\left(\mathbf{H}_{1}\right)$ and relay $\left(\mathbf{H}_{2}\right)$ channel matrices associated with the strongest eigenvalues. This simple result is, however, not valid in the case of AF relaying protocol. Instead, for AF protocol, we propose the use of relay selection scheme to facilitate the design of precoders at the source and relay nodes. Finally, different numerical examples proved that the proposed optimal transmission and reception techniques are indeed effective and provide a meaningful gain in term of received SNR and system capacity while maintaining the complexity very low due to linearity.

\section{References}

[1] P. Gupta and P. R Kumar, "The Capacity of Wireless Networks," IEEE Transactions on Information Theory, Vol. 46, No. 2, March 2002, pp. 388-404.

[2] M. Gastpar and M. Vetterli, "On the Capacity of Wireless Networks: The Relay Case," Proceedings of 21th Annual joint Conference of the IEEE Computer and Communications, New York, Vol. 3, June 2002, pp. 1577-1586.

[3] A. Sendonaris, E. Erkip and B. Aaazhang, "User Cooperation Diversity_Part I: System Description," IEEE Transactions on Communications, Vol. 51, No. 11, November 2003, pp. 1927-1938.

[4] A. Sendonaris, E. Erkip and B. Aaazhang, "User Cooperation Diversity-Part II: Implementation Aspects and Performance Analysis," IEEE Transactions on Communi- cations, Vol. 51, No. 11, November 2003, pp. 1939-1948.

[5] J. N. Laneman, D. N. C. Tse and G. W. Wornell, "Cooperative Diversity in Wireless Networks: Efficient Protocols and Outage Behaviour," IEEE Transactions on Information Theory, Vol. 50, No. 12, December 2004, pp. 3062-3080.

[6] F. H. P. Fitzek and M. D. Katz, "Cooperation in Wireless Networks: Principles and Applications," Springer, Netherland, 2006.

[7] R. U. Nabar, H. Bölcskei and F. W. Kneubuhler, "Fading Relay Channels: Performance Limits and Space-Time Signal Design," IEEE Journal on Selected Areas in Communications, Vol. 22, No. 6, August 2004, pp. 1099- 1109.

[8] J. N. Laneman and G. W. Wornell, "Distributed SpaceTime-Coded Protocols for Exploiting Cooperative Diversity in Wireless Networks," IEEE Transactions on Information Theory, Vol. 49, No. 10, October 2003, pp. 24152425.

[9] A. Stefanov and E. Erkip, "Cooperative Coding for Wireless Networks," IEEE Transactions on Communications, Vol. 52, No. 9, September 2004, pp. 1470-1476.

[10] M. Munoz-Medina, J. Vidal and A. Agustion, "Linear Transceiver Design in Nonregenerative Relays with Channel State Information," IEEE Transactions on Signal Processing, Vol. 55, No. 6, June 2007, pp. 2593-2604.

[11] A. Bletsas, A. Khisti, D. P. Reed and A. Lippman, "A Simple Cooperative Diversity Method Based on Network Path Selection," IEEE Journal on Selected Areas in Communications, Vol. 24, No. 3, March 2006, pp. 659672.

[12] A. Bletsas, "Intelligent Antenna Sharing in Cooperative Diversity Wireless Networks," Ph.D. Dissertation, Massachusetts Institute of Technology, 2005.

[13] H. Bölcskei, R. U. Nabar, O. Oyman and A. J. Paulraj, "Capacity Scaling Laws in MIMO Relay Networks," IEEE Transactions on Wireless Communications, Vol. 5, No. 6, June 2006, pp. 1433-1444.

[14] S. O. Gharan, A. Bayesteh and A. K. Khandani, "Asymptotic Analysis of Amplify and Forward Relaying in a Parallel MIMO Relay Network," submitted for publication. http://cst.uwaterloo.ca

[15] A. Wittneben and B. Rankov, "Impact of Cooperative Relays on the Capacity of Rank-Deficient MIMO Channels," Proceedings of the 12th IST Summin on Mobile Wireless Communications, Aveiro, June 2003, pp. 421-425.

[16] Y. Fan and J. S. Thompson, "MIMO Configurations for Relay Channels: Theory and Practive," IEEE Transactions on Wireless Communications, Vol. 6, No. 5, May 2007, pp. 1774-1786.

[17] X. Tang and Y. Hua, "Optimal Design of Nonregenerative MIMO Wireless Relays," IEEE Transactions on Wireless Communications, Vol. 6, No. 4, April 2007, pp. 1398-1407. 